\title{
The Effects of Different Fungi on the IL-1 $\beta$ Expression in Mouse Dendritic Cells
}

\author{
Li-Juan Zhang ${ }^{1}$, Xiao-Dong Wang ${ }^{1}$, Xue-Feng $\operatorname{Wan}^{1}{ }^{1}$, Hadiliya Hasimu ${ }^{1}$ and Paride Abliz ${ }^{1,}$ \\ ${ }^{1}$ Department of Dermatology, The First Affiliated Hospital of Xinjiang Medical University, Urumqi, China \\ "Corresponding author: Department of Dermatology, The First Affiliated Hospital of Xinjiang Medical University, Urumqi, China. Email: abulizep652871@163.com
}

Received 2020 April 25; Revised 2020 October 28; Accepted 2020 November 06.

\begin{abstract}
Background: Invasive fungal infection received more and more attention because of its high mortality, Candida albicans and Aspergillus fumigatus is the most common pathogenic fungus for systematic fungal infection, A. lentulus was isolated and identified recently and named as a sister of A. fumigatus.

Objectives: The current study aimed to explore the concentration and time-dependent relationships of the virulence of fungi due to the change in the interleukin-1 beta (IL-1 $\beta$ ) level.

Methods: Candida albicans, A. fumigatus, and A. lentulus suspensions with a multiplicity of infection $=0,1,5,10$, and 20 units were used to treat mouse dendritic cells. The IL- $\beta$ level was measured by enzyme-linked immunosorbent assay (ELISA) at 2, 6, 12, 24, and 48 hours after the treatment was administered.

Results: The main effects and interactions between the multiplicity of infection, intervention duration, and the dependent variable of IL-1 $\beta$ were significant. Besides, there were statistically significant differences. Only C. albicans and $A$. lentulus could induce IL-1 $\beta$ 48 hours after administration. Furthermore, the production of IL-1 $\beta$ induced by $A$. fumigatus was higher than that induced by $A$. lentulus and C. albicans.

Conclusions: This study demonstrated concentration- and time-dependent relationships in IL-1 $\beta$ production by dendritic cells induced by three types of fungi. Candida albicans and A. lentulus exhibited a slow phase-in in vitro inflammation induction. The inflammatory response induced by A. fumigatus in vitro has the characteristics of a short action time and a strong toxic effect. Finally, $A$. lentulus is less virulent than A. fumigatus, and its inflammation-inducing time is relatively longer.
\end{abstract}

Keywords: Candida albicans, Aspergillus fumigatus, A. lentulus, IL-1 $\beta$

\section{Background}

The incidence of opportunistic fungal infections is on the rise, mainly due to population aging and enhanced incidence of malignant tumors. Candida is the most common cause of digestive tract infection, while Aspergillus is known as the most likely cause of invasive lung infection (1). In China, the mortality of untreated pulmonary Aspergillus infection is high (2-4), and Aspergillus fumigatus infections account for $70-80 \%$ of pulmonary Aspergillus infections (5). Recent developments in molecular-based approaches have resulted in detecting new species of Aspergillus (6). These rare Aspergillus are different from A. fumigatus in toxicology, drug resistance, and growth conditions (6-8). Aspergillus lentulus was first isolated and identified in patients with leukemia by Balajee in 2005 and named as a sister of A. fumigatus (9).

Subsequently, scholars could isolate A. lentulus from bronchoalveolar lavage fluid and sputum samples of patients who underwent heart, liver, and kidney transplantations and patients with chronic obstructive pulmonary emphysema and cystic fibrosis in Japan (10), Brazil (11), Switzerland (12), Spain (13), Argentina (14), Turkey (15), and other countries. Therefore, it has attracted more attention over the years. In 2011, our team isolated A. lentulus from the sputum of an elderly male patient who had chronic pulmonary heart disease and acute pulmonary infection for 5 years (16). Finally, the patient died of invasive pulmonary fungal infection despite receiving active treatment. This was the first time that Chinese scholars could find cases of A. lentulus infection and cases of non-immune deficiency infection in China. However, the virulence and host im- 
mune response pathway of the fungus remain unclear.

Natural immunity is the most important line of defense against fungal infections, and the NACHT, LRR, and PYD domains-containing protein 3 (NLRP3) inflammatory corpuscle pathway plays a crucial in fungus-induced host immune response. Fungi can induce the NLRP3 inflammatory pathway, which in turn causes releasing inflammatory factors by infected cells, such as tumor necrosis factor$\alpha$ (TNF- $\alpha$ ) and interleukin-1 beta (IL-1 $\beta$ ), to resist against fungal invasion. IL-1 $\beta$ is an important immunomodulator against infection and injury in the NLRP3 pathway. It has also been reported that IL-1 $\beta$ is required for the host to inhibit Candida albicans infection (16-19).

\section{Objectives}

The current study aimed to investigate the ability of three fungi (i.e. C. albicans, A. fumigatus, and A. lentulus) to induce the host immune response of mouse dendritic cells by detecting the production of IL-1 $\beta$.

\section{Methods}

The experimental cells that included DC2.4 mouse dendritic cells, a cell type, were cultured in RPMI-1640 medium $+10 \%$ fetal bovine serum $+1 \%$ phosphate-buffered saline (PBS) with $5 \% \mathrm{CO} 2$ at $37^{\circ} \mathrm{C}$ and saturated humidity (5). The experimental fungi were C. albicans, A. fumigatus, and $A$. lentulus.

\subsection{The Cell Cultures and Fungal Cultures}

The frozen cells were taken out of the liquid nitrogen and immediately placed in a $37^{\circ} \mathrm{C}$ water bath, and the cryopreservation tube was shaken quickly. Then, the cells were thawed quickly for two minutes. Furthermore, $9 \mathrm{~mL}$ of complete medium was added to a $15 \mathrm{~mL}$ centrifuge tube in advance. The thawed cells were quickly added to the 15 $\mathrm{mL}$ centrifuge tube and then centrifuged, and the supernatant was discarded. The cells were transferred to a culture flask, cultured at $37^{\circ} \mathrm{C}$ with saturated humidity and $5 \%$ $\mathrm{CO}_{2}$ in an incubator, and sub-cultured for future use. The DC2.4 cells, with a good confluence rate of $90 \%$, were taken, trypsinized, and passaged at a ratio of 1:4 to the 7th day.

Lipopolysaccharide with a final concentration of 1 $\mathrm{mg} / \mathrm{L}$ was added for 48 hours. Afterward, the suspended cells were collected and passaged at a ratio of 1:3, and the cell suspension was collected before the experiment. Using Sabouraud medium, C. albicans and A. fumigatus were cultured at $37^{\circ} \mathrm{C}$ for 4 days, and $A$. lentulus was cultured at $37^{\circ} \mathrm{C}$ for 9 days (6). The selected boll glucose agar was produced by Becton, Dickinson and Company (New York, NJ, USA, date of production: 2017-02, a period of validity: 202201, batch number of production: 7109972). When used, 19.5 $\mathrm{g}$ of prepared medium powder was dissolved in $500 \mathrm{~mL}$ of distilled water. After full dissolution, the pressure cooker was disinfected and sterilized. The powder was placed in a MEA $9 \mathrm{~cm}$ petri dish on the sterile operating table and stored at low temperature.

The cell concentration was adjusted to $5 \times 10^{4}$ cells $/ \mathrm{mL}$ with complete medium (10\% serum RPMI-1640 complete medium: $10 \mathrm{~mL}$ of fetal bovine serum was added to 90 $\mathrm{mL}$ of RPMI-1640 medium, and $1 \mathrm{~mL}$ of a dual-antibody solution of penicillin-streptomycin was added. The concentration of the cell suspension was adjusted to $5 \times 10^{4}$ cells/mL using complete medium) and the cells were inoculated into a 24-well plate, $500 \mathrm{uL} /$ well. The cultured C. albicans, A. fumigatus, and A. lentulus were grinded and then rinsed with sterile PBS three times, counted using a counting board, and then the DC2.4 cells were infected with these fungi with a multiplicity of infection $=0,1,5,10$, and 20 units. After 2, 6, 12, 24, and 48 hours of infection, the supernatant was collected.

\subsection{The Detection of IL-1 $\beta$}

In strict accordance with the instructions of the mouse IL-1 $\beta$ enzyme-linked immunosorbent assay (ELISA) kit (MULTI SCIENCES, Hangzhou, China), the supernatant of the cell culture medium of the above groups was detected by ELISA.

\subsection{Statistical Analysis}

Data were analyzed using SPSS version 19.0 (International Business Machines Corporation, New York, America). Data are expressed as mean \pm standard deviation (X $\pm \mathrm{SD}$ ). Initially, multivariate analysis of variance (ANOVA) was used to analyze the correlation between the main effect, the interaction effect, and the dependent variables. Then, univariate ANOVA was performed. If the data were normally distributed, univariate ANOVA was used. Data were analyzed by multiple comparisons using the least significant difference method, and data with heterogeneity of variance underwent multiple comparisons using the Tamhane method. Statistical significance was considered when P-value $<0.05$. For skewed data, $\log$ transformation was used to approximately conform to normality. Then, the ANOVA method was used to analyze the data. 


\section{Results}

4.1. The Analysis of the Relationship Between the Production of IL-1 $\beta$ Induced by Three Types of Fungi and Their Action Time and Concentration

The main effects and interactions among the multiplicity of infection value, intervention duration, and the dependent variable IL-1 $\beta$ among C. albicans, A. fumigatus, and A. lentulus were significant, and the differences were statistically significant (Tables 1-3). Based on the findings, action time and multiplicity of infection value contributed to the increase in IL-1 $\beta$ induced by the three types of fungi. Besides, we found an interaction between them.

4.2. The Two-Factor Analysis of IL-1 $\beta$ Production by Three Types of Fungi with Different Multiplicity of Infection Values at Different Intervention Times

As mentioned before, the IL- $1 \beta$ production induced by the three types of fungi was related to the action time and concentration. Thereafter, a two-factor analysis of IL-1 $\beta$ production by three types of fungi with different multiplicity of infection values at different intervention times was performed (Figures 1 and 2 ). The increase in IL-1 $\beta$ could only be induced by $C$. albicans after 48 hours of treatment and by A. fumigatus after 12 hours of treatment; the highest level of toxicity was observed after 48 hours, and multiplicity of infection was one unit. Similar to C. albicans, the increase in IL-1 $\beta$ could only be induced by $A$. lentulus after 48 hours of treatment; however, in contrast to C. albicans, it induced a significant increase when multiplicity of infection was one, that is, the inflammatory effect of A. lentulus was stronger than that of C. albicans.

4.3. The Analysis of the Difference in IL-1 $\beta$ Among the Three Groups at the Same Time and the Same Multiplicity of Infection

Finally, to determine the magnitude of the inflammation-inducing effect of the three types of fungi, the difference in IL-1 $\beta$ level among the three groups at the same time and the same multiplicity of infection was analyzed. After treating the three groups with the mouse dendritic cells at 12 hours, the IL-1 $\beta$ level was increased significantly, and at 12,24 , and 48 hours, the IL-1 $\beta$ level was higher in the A. fumigatus treated group than the $C$. albicans and $A$. lentulus groups, and the differences were statistically significant. The highest level of IL-1 $\beta$ was found at 48 hours in all three groups; however, the best multiplicity of infection value was different: multiplicity of infection $=20$ in C. albicans, multiplicity of infection
$=1$ in A. fumigatus, and multiplicity of infection $=5$ in $A$. lentulus. At the same time and with similar value of multiplicity of infection, the inflammation-inducing effect of A. fumigatus was always greater than that of A. lentulus. In the $C$. albicans group, the inflammation-inducing effect was continuously and positively correlated with time and concentration.

\section{Discussion}

Candida is the most common cause of digestive tract infection, and Aspergillus is an invasive lung infection caused by Aspergillus (1). Moreover, in recent years, new species of Aspergillus are reported; however, their toxicology, drug resistance, and growth conditions have not been fully defined. A. lentulus is a new Aspergillus that was identified in 2005 by Balajee and gradually attracted researchers' attention (11). However, still the virulence and host immune response pathway of this fungus are unclear. To a certain extent, virulence contributes to the inflammatory response induced by fungus. In this study, the level of IL- $\beta$ after the action of C. albicans, A. fumigatus, and A. lentulus on the mouse dendritic cells was measured to indirectly reflect the inflammation-inducing effect and virulence of the three types of fungi.

This study demonstrated that the production of IL$1 \beta$ induced by the three fungi depended on their concentration and action duration, where the inflammationinducing effect of $C$. albicans presented significant concentration and time-dependent patterns. Its virulence is weaker than that of A. fumigatus, because C. albicans can stimulate the NLRP3 activation only when it changes from the yeast phase to the mycelium phase (19-22). A previous study on the host immune response of A. fumigatus revealed that the IL-1 $\beta$ level was significantly increased in human dendritic cells 6 hours after being treated with the fragment of A. fumigatus; therefore, A. fumigatus had the characteristic of fast inflammation (23).

This finding is consistent with the finding of our study. Aspergillus lentulus is a new strain, and sufficient evidence about its virulence and host immune response are not available. It's well-documented that A. lentulus can infect not only immunocompromised patients but also patients with non-granulocytopenia, and the disease has a very high mortality rate. The present study revealed that the inflammation-inducing effect of $A$. lentulus was weaker than that of $A$. fumigatus at the same concentration and time, and its high mortality rate can be attributed to its 


\begin{tabular}{|c|c|c|c|c|c|}
\hline Items & III-Type Quadratic Sum & df & Mean Square & $\mathbf{F}$ & Sig. \\
\hline Correction model & $2271.938^{\mathrm{a}}$ & 24 & 94.664 & 45.226 & 0.000 \\
\hline Nodal increment & 8306.677 & 1 & 8306.677 & 3968.494 & 0.000 \\
\hline Multiplicity of infection & 449.367 & 4 & 112.342 & 53.671 & 0.000 \\
\hline Intervention durations & 410.549 & 4 & 102.637 & 49.035 & 0.000 \\
\hline Multiplicity of infection * intervention durations & 1412.022 & 16 & 88.251 & 42.162 & 0.000 \\
\hline Error & 104.658 & 50 & 2.093 & & \\
\hline Total & 10683.273 & 75 & & & \\
\hline Corrected total & 2376.596 & 74 & & & \\
\hline
\end{tabular}

${ }^{\mathrm{a}} \mathrm{R}$ square $=0.956($ adjusted $\mathrm{R}$ square $=0.935)$.

\begin{tabular}{lccccc}
\hline Table 2. Intersubjective Effect for Aspergillus fumigatus & & & & & \\
\hline Items & III-Type Quadratic Sum & df & Mean Square & F & Sig. \\
\hline Correction model & $17314.092^{\mathrm{a}}$ & 24 & 721.421 & 261.855 & 0.000 \\
\hline Nodal increment & 22496.117 & 1 & 22496.117 & 8165.453 & 0.000 \\
\hline Multiplicity of infection & 1444.550 & 4 & 361.138 & 131.083 & 0.000 \\
\hline Intervention durations & 9927.632 & 4 & 2481.908 & 900.862 & 0.000 \\
\hline Multiplicity of infection * intervention durations & 5941.910 & 16 & 371.369 & 134.797 & 0.000 \\
\hline Error & 137.752 & 50 & 2.755 & & \\
\hline Total & 39947.961 & 75 & & &
\end{tabular}

${ }^{\mathrm{a}} \mathrm{R}$ square $=0.992($ adjusted $\mathrm{R}$ square $=0.988)$.

Table 3. Intersubjective Effect for Aspergillus lentulus

\begin{tabular}{lccccc}
\hline Items & III-Type Quadratic Sum & df & Mean Square & F & Sig. \\
\hline Correction model & $1756.644^{\mathrm{a}}$ & 24 & 73.194 & 57.040 & 0.000 \\
\hline Nodal increment & 8374.738 & 1 & 8374.738 & 6526.471 & 0.000 \\
\hline Multiplicity of infection & 194.161 & 4 & 48.540 & 37.828 & 0.000 \\
\hline Intervention durations & 959.601 & 4 & 239.900 & 186.955 & 0.000 \\
\hline Multiplicity of infection ${ }^{*}$ intervention durations & 602.882 & 16 & 37.680 & 29.364 & 0.000 \\
\hline Error & 64.160 & 50 & 1.283 & \\
\hline Total & 10195.542 & 75 & & & \\
Corrected total & 1820.804 & 74 & & &
\end{tabular}

${ }^{\mathrm{a}} \mathrm{R}$ square $=0.965($ adjusted $\mathrm{R}$ square $=0.948)$.

high resistance to currently available drugs. This is consistent with the results of previous studies and our previous drug sensitivity tests (23).

Some scholars have used the larvae infection model to investigate this issue, and reported that A. fumigatus was more virulent than $A$. lentulus. In these previously conducted studies, two days after the infection, the active mycelial growth of A. fumigatus was not visible in the lar- 

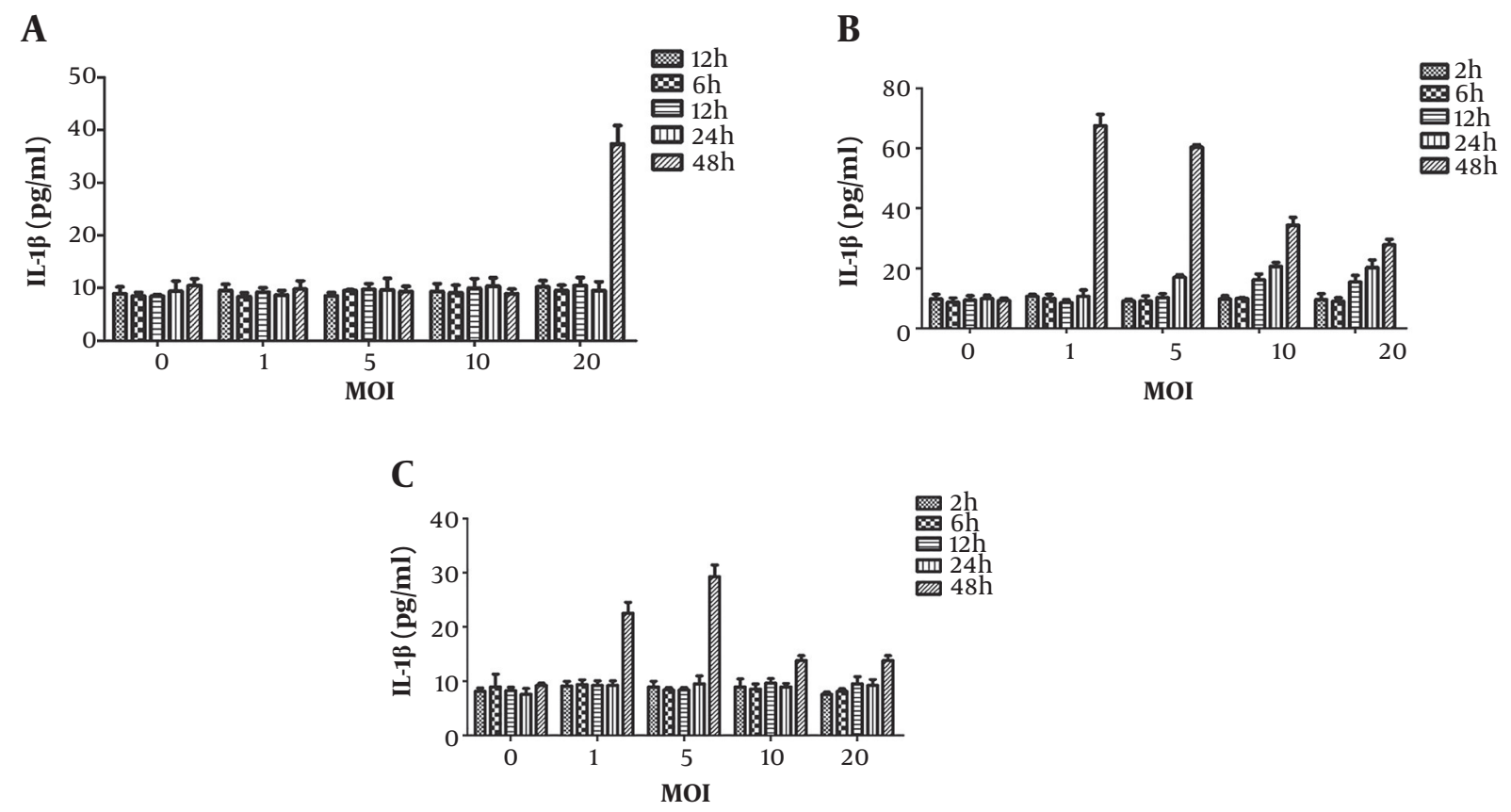

Figure 1. The effect of different fungi with different intervention durations on the level of IL-1 $\beta$ in the supernatant of DC mouse dendritic cells. A, The effect of Candida with different multiplicity of infection values and different intervention durations on the level of IL-1 $\beta$ in the supernatant of DC mouse dendritic cells; B, the effect of Aspergillus fumigatus with different multiplicity of infection values and different intervention durations on the level of IL-1 $\beta$ in the supernatant of DC mouse dendritic cells; $C$, the effects of $A$. lentulus with different multiplicity of infection values and different intervention durations on the level of IL-1 $\beta$ in the supernatant of DC mouse dendritic cells. MOI, multiplicity of infection; DC, dendritic cells.

vae tissue section, whereas A. lentulus grew slowly and was surrounded by the adjacent tissue cells (20). In this in vitro study, compared to A. fumigatus, A. lentulus showed a shorter duration of inflammation, which further supports the fact that $A$. lentulus belongs to slow phase fungi and its multidrug resistance may be an important factor for its poor prognosis. However, further studies are needed to prove this hypothesis. Moreover, further studies on different cells and animal models are needed to investigate relevant mechanisms.

\subsection{Conclusions}

There are concentration and time-dependent relationships in IL-1 $\beta$ production by dendritic cells induced by $A$. fumigatus, C. albicans, and A. lentulus. Besides, A. lentulus exhibited a slow phase in in vitro inflammation induction. The inflammatory response induced by A. fumigatus in vitro has the characteristics of a short action time and a strong toxic effect. Finally, A. lentulus is less virulent than A. fumigatus and the inflammation-inducing time is relatively longer.

\section{Acknowledgments}

We would like to acknowledge the hard and dedicated work of all the staff that implemented the intervention and evaluation components of the study.

\section{Footnotes}

Authors' Contribution: Li-Juan Zhang and Paride Abliz did substantial contributions to the conception and design of the work, and draft the work. Li-Juan Zhang, XiaoDong Wang, Xue-Feng Wan, Hadiliya Hasimu, and Paride Abliz did the acquisition, analysis, and interpretation of data for the work. Li-Juan Zhang, Xiao-Dong Wang, XueFeng Wan, Hadiliya Hasimu, and Paride Abliz did revising it critically for important intellectual content. Li-Juan Zhang, Xiao-Dong Wang, Xue-Feng Wan, Hadiliya Hasimu, and Paride Abliz did final approval of the version to be published. Li-Juan Zhang, Xiao-Dong Wang, Xue-Feng Wan, Hadiliya Hasimu, and Paride Abliz did agreement to be accountable for all aspects of the work in ensuring that questions related to the accuracy or integrity of any part of the work are appropriately investigated and resolved. 
A

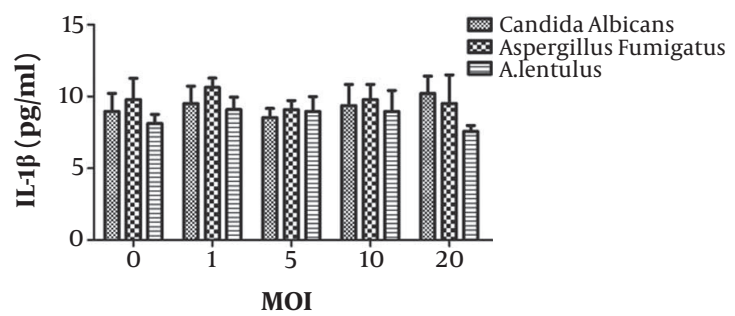

C

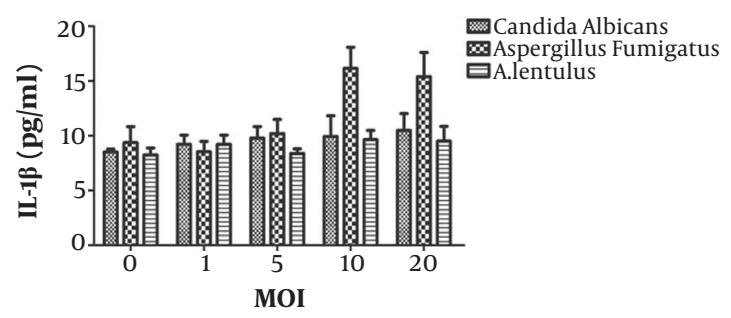

B

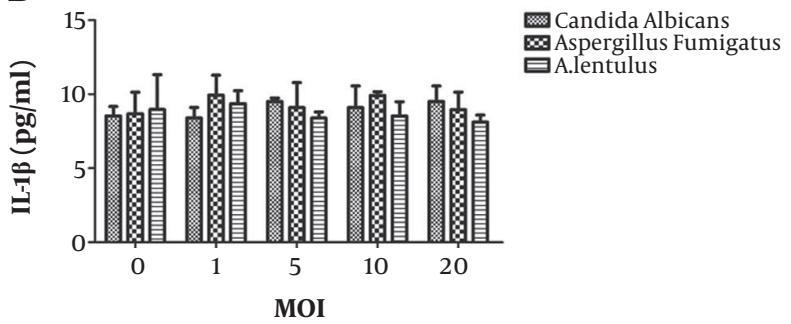

D

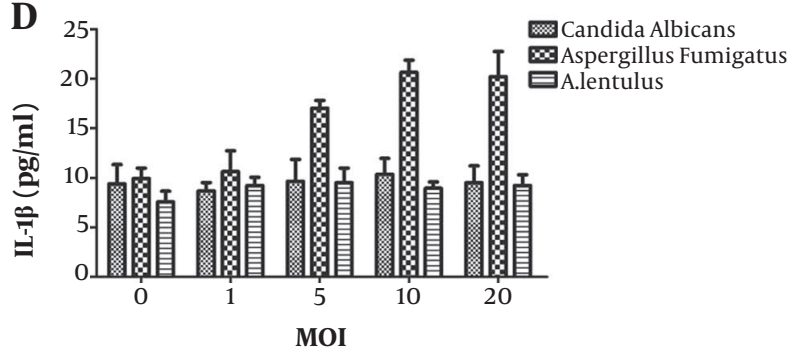

E

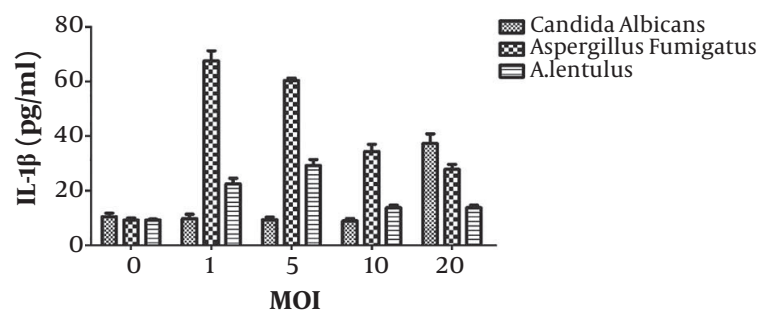

Figure 2. The effect of different fungi with different intervention durations on the level of IL-1 $\beta$ in the supernatant of DC mouse dendritic cells. A, The effect of different fungi with 2 hours of intervention on the level of IL-1 $\beta$ in the supernatant of DC mouse dendritic cells; B, the effect of different fungi with 6 hours of intervention on the level of IL-1 $\beta$ in the supernatant of DC mouse dendritic cells; $C$, the effect of different fungi with 12 hours of intervention on the level of IL-1 $\beta$ in the supernatant of DC mouse dendritic cells; D, the effect of different fungi with 24 hours of intervention on the level of IL-1 $\beta$ in the supernatant of DC mouse dendritic cells; E, the effect of different fungi with 48 hours of intervention on the level of IL-1 $\beta$ in the supernatant of DC mouse dendritic cells. MOI, multiplicity of infection; DC, dendritic cells.

Conflict of Interests: The authors declare no conflict of interest.

Ethical Approval: This study is approved by the Ethics Committee of The First Affiliated Hospital of Xinjiang Medical University.

Funding/Support: This project was supported by the National Natural Science Foundation of China (grant no.: 81760360).

\section{References}

1. Mirhendi H, Charsizadeh A, Eshaghi H, Nikmanesh B, Arendrup MC. Species distribution and antifungal susceptibility profile of Candida isolates from blood and other normally sterile foci from pediatric ICU patients in Tehran, Iran. Med Mycol. 2020;58(2):201-6. doi: 10.1093/mmy/myz047. [PubMed: 31111910].

2. Zarrinfar H, Kaboli S, Dolatabadi S, Mohammadi R. Rapid detection of Candida species in bronchoalveolar lavage fluid from pa- tients with pulmonary symptoms. Braz J Microbiol. 2016;47(1):172-6. doi: 10.1016/j.bjm.2015.02.001. [PubMed: 26887241]. [PubMed Central: PMC4822774].

3. Zarrinfar H, Makimura K, Satoh K, Khodadadi H, Mirhendi H. Incidence of pulmonary aspergillosis and correlation of conventional diagnostic methods with nested PCR and real-time PCR assay using BAL fluid in intensive care unit patients. J Clin Lab Anal. 2013;27(3):1815. doi: 10.1002/jcla.21580. [PubMed: 23686776]. [PubMed Central: PMC6807532].

4. Beck J, Broniszewska M, Schwienbacher M, Ebel F. Characterization of the Aspergillus fumigatus chitosanase CsnB and evaluation of its potential use in serological diagnostics. Int J Med Microbiol. 2014;304(56):696-702. doi: 10.1016/j.ijmm.2014.05.001. [PubMed: 24880790].

5. Barchiesi F, Mazzocato S, Mazzanti S, Gesuita R, Skrami E, Fiorentini A, et al. Invasive aspergillosis in liver transplant recipients: epidemiology, clinical characteristics, treatment, and outcomes in 116 cases. Liver Transpl. 2015;21(2):204-12. doi: 10.1002/lt.24032. [PubMed: 25348192].

6. Zanganeh E, Zarrinfar H, Rezaeetalab F, Fata A, Tohidi M, Najafzadeh $\mathrm{MJ}$, et al. Predominance of non-fumigatus Aspergillus species among 
patients suspected to pulmonary aspergillosis in a tropical and subtropical region of the Middle East. Microb Pathog. 2018;116:296-300. doi: 10.1016/j.micpath.2018.01.047. [PubMed: 29410233].

7. Zarei F, Mirhendi H, Motamedi M, Ahmadi B, Nouripour-Sisakht S, Zarrinfar $\mathrm{H}$, et al. Black Aspergillus species isolated from clinical and environmental samples in Iran. J Med Microbiol. 2015;64(11):1454-6. doi:10.1099/jmm.0.000166. [PubMed: 26384733].

8. Tavakoli M, Rivero-Menendez O, Abastabar M, Hedayati MT, Sabino $\mathrm{R}$, Siopi M, et al. Genetic diversity and antifungal susceptibility patterns of Aspergillus nidulans complex obtained from clinical and environmental sources. Mycoses. 2020;63(1):78-88. doi: 10.1111/myc.13019. [PubMed: 31609022].

9. Balajee SA, Gribskov JL, Hanley E, Nickle D, Marr KA. Aspergillus lentulus sp. nov., a new sibling species of A. fumigatus. Eukaryot Cell. 2005;4(3):625-32. doi: 10.1128/EC.4.3.625-632.2005. [PubMed: 15755924]. [PubMed Central: PMC1087803].

10. Yoshida H, Seki M, Umeyama T, Urai M, Kinjo Y, Nishi I, et al. Invasive pulmonary aspergillosis due to Aspergillus lentulus: Successful treatment of a liver transplant patient.JInfect Chemother. 2015;21(6):479-81. doi: 10.1016/j.jiac.2015.02.010. [PubMed: 25828927].

11. Bastos VR, Santos DW, Padovan AC, Melo AS, Mazzolin Mde A, Camargo LF, et al. Early invasive pulmonary aspergillosis in a kidney transplant recipient caused by Aspergillus lentulus: first Brazilian report. Mycopathologia.2015;179(3-4):299-305. doi:10.1007/s11046-014-9840-7. [PubMed: 25515242].

12. Zbinden A, Imhof A, Wilhelm MJ, Ruschitzka F, Wild P, Bloemberg GV, et al. Fatal outcome after heart transplantation caused by Aspergillus lentulus. Transpl Infect Dis. 2012;14(5):E60-3. doi: 10.1111/j.1399-3062.2012.00779.x. [PubMed: 22988985].

13. Alhambra A, Catalan M, Moragues MD, Brena S, Ponton J, Montejo $\mathrm{JC}$, et al. Isolation of Aspergillus lentulus in Spain from a critically ill patient with chronic obstructive pulmonary disease. Rev Iberoam Micol.2008;25(4):246-9. doi:10.1016/s1130-1406(08)70058-5. [PubMed: 19071895].

14. Montenegro G, Sanchez Puch S, Jewtuchowicz VM, Pinoni MV, Relloso S, Temporitti E, et al. Phenotypic and genotypic characterization of Aspergillus lentulus and Aspergillus fumigatus isolates in a patient with probable invasive aspergillosis. J Med Microbiol. 2009;58(Pt 3):391-5. doi: 10.1099/jmm.0.005942-0. [PubMed: 19208894].
15. Gurcan S, Tikvesli M, Ustundag S, Ener B. A Case Report on Aspergillus lentulus Pneumonia. Balkan Med J. 2013;30(4):429-31. doi: 10.5152/balkanmedj.2013.8572. [PubMed: 25207153]. [PubMed Central: PMC4115954].

16. Vonk AG, Netea MG, van Krieken JH, Iwakura Y, van der Meer JW, Kullberg BJ. Endogenous interleukin (IL)-1 alpha and IL-1 beta are crucial for host defense against disseminated candidiasis. J Infect Dis. 2006;193(10):1419-26. doi: 10.1086/503363. [PubMed:16619190].

17. Joly S, Ma N, Sadler JJ, Soll DR, Cassel SL, Sutterwala FS. Cutting edge: Candida albicans hyphae formation triggers activation of the Nlrp3 inflammasome. J Immunol. 2009;183(6):3578-81. doi: 10.4049/jimmunol.0901323. [PubMed: 19684085]. [PubMed Central: PMC2739101].

18. Joly S, Sutterwala FS. Fungal pathogen recognition by the NLRP3 inflammasome. Virulence. 2010;1(4):276-80. doi: 10.4161/viru.1.4.11482. [PubMed: 21178453].

19. Hise AG, Tomalka J, Ganesan S, Patel K, Hall BA, Brown GD, et al. An essential role for the NLRP3 inflammasome in host defense against the human fungal pathogen Candida albicans. Cell Host Microbe. 2009;5(5):487-97. doi: 10.1016/j.chom.2009.05.002. [PubMed: 19454352]. [PubMed Central: PMC2824856].

20. Gross O, Poeck H, Bscheider M, Dostert C, Hannesschlager N, Endres $S$, et al. Syk kinase signalling couples to the Nlrp3 inflammasome for anti-fungal host defence. Nature. 2009;459(7245):433-6. doi: 10.1038/nature07965. [PubMed: 19339971].

21. Kankkunen P, Teirila L, Rintahaka J, Alenius H, Wolff H, Matikainen S. (1,3)-beta-glucans activate both dectin-1 and NLRP3 inflammasome in human macrophages. J Immunol. 2010;184(11):6335-42. doi: 10.4049/jimmunol.0903019. [PubMed: 20421639].

22. Kumar H, Kumagai Y, Tsuchida T, Koenig PA, Satoh T, Guo Z, et al. Involvement of the NLRP3 inflammasome in innate and humoral adaptive immune responses to fungal beta-glucan. $J$ Immunol. 2009;183(12):8061-7. doi: 10.4049/jimmunol.0902477. [PubMed: 20007575].

23. Said-Sadier N, Padilla E, Langsley G, Ojcius DM. Aspergillus fumigatus stimulates the NLRP3 inflammasome through a pathway requiring ROS production and the Syk tyrosine kinase. PLoS One. 2010;5(4). e10008. doi: 10.1371/journal.pone.0010008. [PubMed: 20368800]. [PubMed Central: PMC2848854]. 